\title{
Establishing a National Syndromic Surveillance System among Asylum Seekers
}

\author{
Mariette Hooiveld $^{\star 1,2}$, Madelief Mollers ${ }^{2}$, Stephanie van Rooden ${ }^{1}$, Robert A. Verheij' and \\ Susan J. Hahné ${ }^{2}$
}

${ }^{1}$ NIVEL, Utrecht, Netherlands; ${ }^{2}$ RIVM, Centre for Infectious Disease Control, Bilthoven, Netherlands

\section{Objective}

Facing challenges to establish a new national syndromic surveillance system in the Netherlands for infectious diseases among asylum seekers.

\section{Introduction}

Most European countries are facing a continuous increased influx of asylum seekers [1]. Poor living conditions in crowded shelters and refugee camps increase the risk for - outbreaks of - infectious diseases in this vulnerable population. In line with ECDC recommendations [2], we aim to improve information on infectious diseases among asylum seekers by establishing a new syndromic surveillance system in the Netherlands. This system will complement the notifiable disease system for infectious diseases.

The aim of the syndromic surveillance system is to improve the detecting of outbreaks of infectious diseases in asylum seekers' centres in an early stage of development to be able to take adequate and timely measures to prevent further spread, and to collect information on the burden of infection within this population.

\section{Methods}

Primary health care for asylum seekers in the Netherlands is organized nationally by the Asylum Seekers Health Centre, with general practitioners providing care in each reception centre. General practitioners (GPs) act as gatekeepers for specialized, secondary health care and the GP is the first professional to consult for health problems. Therefore, electronic health records (EHR) kept by GPs provide a complete picture of this population. These EHRs contain data on diagnoses/symptoms and treatment of asylum seekers, using the International Classification of Primary Care (ICPC). This data is recorded routinely, as part of the health care process. During summer 2016, about 30,000 asylum seekers were housed in about 60 reception centres across the Netherlands.

\section{Results}

The governance structure was layed down in a collaboration agreement between the Asylum Seekers Health Centre, the national institute of public health RIVM and NIVEL. To ensure privacy of the asylum seekers, a privacy protocol has been drawn, taking into account strict privacy regulations in the Netherlands. The information system provider of the health care centre developed an extraction tool that automatically generates weekly data extracts from the electronic health records system to a Trusted Third Party (TTP). Before transferring the data to NIVEL, the TTP removes directly identifying patient information, indirectly identifying information like date of birth is replaced by quarter and year, and the personal identification number is replaced by a pseudonym. At NIVEL, all data is stored in a relational database, from which weekly research extracts are generated for infectious disease surveillance at RIVM after applying a second pseudonymisation step (two-way pseudonimisation) [3]. First data extracts are being expected mid-October 2016, after which data quality will be evaluated. Weekly, or daily, consultations rates will be calculated based on the number of cases meeting predefined definitions, stratified by immigration centre, age group, sex and nationality. Numerators will be based on the number of population housed in the immigration centres.

\section{Conclusions}

With the cooperation of a national health care centre, providing primary care to asylum seekers housed at several locations, and the information system provider of the health care centre, EHRs can be used for syndromic surveillance, taking into account strict privacy regulations. The new surveillance system will be evaluated after one year, focusing on data quality, usefulness, and the added value above to the notification of diseases.

\section{Keywords}

Asylum seekers; Syndromic surveillance; Electronic Health Records; Governance

\section{References}

1. Catchpole M, Coulombier D. Refugee crisis demands European Unionwide surveillance! Euro Surveill 2015,20.

2. European Centre for Disease Prevention and Control (ECDC). Communicable disease risks associated with the movement of refugees in Europe during the winter season. Rapid risk assessment. Stockholm: ECDC; 2015.

3. Kuchinke W, Ohmann C, Verheij RA, van Veen EB, Delaney B. Development towards a learning health system - experiences with the privacy protection model of the TRANSFoRm project. In: Gutwirth S, et al (eds). Data protection on the move. Springer, Dordrecht: 2016.

\section{*Mariette Hooiveld}

E-mail:m.hooiveld@nivel.nl 